International Journal of Pure and Applied Mathematics

Volume 94 No. 2 2014, 119-131

ISSN: 1311-8080 (printed version); ISSN: 1314-3395 (on-line version)

url: http://www.ijpam.eu

doi: http://dx.doi.org/10.12732/ijpam.v94i2.1

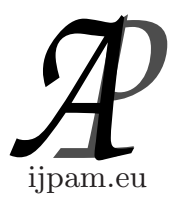

\title{
SOLUTION OF THE TWO-DIMENSIONAL SECOND-ORDER DIFFUSION EQUATION WITH NONLOCAL BOUNDARY CONDITION
}

\author{
Samaneh Afshar ${ }^{1}$, B. Soltanalizadeh ${ }^{2} \S$ \\ ${ }^{1}$ Department of Mathematics \\ Islamic Azad University \\ Central Tehran Branch \\ Tehran, IRAN \\ ${ }^{2}$ Department of Mathematics \\ University of Houston \\ 4800 Calhoun Rd, Houston, TX 77204, USA
}

\begin{abstract}
In the mathematical modeling of many physical phenomena, the diffusion equations with nonlocal boundary condition can be appeared. In this paper, we focus on the two-dimensional inhomogeneous diffusion equations subject to a nonlocal boundary condition. We transform the model of partial differential equation (PDE) into a system of first order, linear, ordinary differential equations (ODEs).
\end{abstract}

AMS Subject Classification: $35 \mathrm{~K} 05,35 \mathrm{~K} 30$

Key Words: diffusion equations, nonlocal boundary conditions, parabolic PDEs, expansion methods, differential transformation method

\section{Introduction}

The partial differential equations with supplementary conditions are one of the

Received: August 29, 2013

(c) 2014 Academic Publications, Ltd.

$\S$ Correspondence author url: www.acadpubl.eu 
most important branches of the applied sciences and many authors paid much attention to solving this case of equations.

In the last decades, the development of numerical methods for the solution of nonlocal boundary value problems has been an important research area in many branches of science such as chemical diffusion, thermoelasticity, heat conduction processes, population dynamics, inverse problems, control theory and certain biological processes. For example, certain chemicals absorb light at various frequencies can be formulated at the special Heat equation with a nonlocal boundary condition. In [28] an example from meteorology for the evolution of the temperature distribution of air near the ground during calm clear nights has given. The other special partial differential equations with nonlocal boundary condition describe the quasi-static flexure of a thermoelastic rod of unit length[29] and the entropy per unit volume[30].

In 1963, Cannon [8] and Batten [9], discussed on the nonlocal boundary equation independently. thereafter, Kamynin [2] and Ionkin [3] investigated parabolic initial-boundary problems with integral conditions for parabolic equations. Beilin [6] investigated the non-local analogue to classical mixed problems, which involve initial, boundary integral conditions. Gordeziani and Avalishvili [7] discussed hyperbolic equations with non-local boundary conditions. A new matrix formulation technique with arbitrary polynomial bases has been proposed for the numerical/analytical solution of the Heat [1] and Telegraph with nonlocal boundary condition and Two matrix formulation techniques based on the shifted standard and shifted Chebyshev bases are proposed for the numerical solution of the wave equation with the non-local boundary condition [10].

There are some papers that discussed the two-dimensional parabolic partial differential equations with nonlocal boundary conditions and Dirichlet boundary conditions $[32,33]$. In this paper we focus on the following diffusion equation in two space variables

$$
u_{t}-\alpha u_{x x}-\beta u_{y y}=f(x, y, t), 0<x<1,0<y<1, t>0,
$$

while the initial condition is

$$
u(x, y, 0)=p(x, y), 0 \leq x \leq 1,0 \leq y \leq 1,
$$

and the dirichlet time-dependent boundary conditions are assumed to be of the form

$$
\begin{aligned}
& u(0, y, t)=q(y, t), 0 \leq t \leq T, 0 \leq y \leq 1, \\
& u(x, 0, t)=h(x, t), 0 \leq t \leq T, 0 \leq x \leq 1, \\
& u(1, y, t)=r(y, t), 0 \leq t \leq T, 0 \leq y \leq 1,
\end{aligned}
$$




$$
u(x, 1, t)=g(x, t), 0 \leq t \leq T, 0 \leq x \leq 1,
$$

and with the nonlocal boundary condition

$$
\int_{0}^{b} \int_{0}^{b^{\prime}} u(x, y, t) d x d y=m(t), 0 \leq x \leq 1,0 \leq y \leq 1 .
$$

The functions $f(x, t), p(x, y), q(x, t), h(x, t), r(y, t), g(x, t)$ and $m(t)$ are known functions and the constants $\alpha, \beta, a$ and $b$ are known constants.

M. Siddique presented Pade schemes [31] and a third order $L_{0}$-stable numerical scheme [27] for the numerical solution of problem (1)-(7). Authors of $[24,25,26]$, proposed some numerical solution to the (1)-(7). The method is based on finding a solution in the form of a polynomial in three variables $U_{n}(x, y, t)=\sum_{i=0}^{n} \sum_{j=0}^{n} \sum_{k=0}^{n} U(i, j, k) x^{i} y^{j} t^{k}$ with undetermined coefficients $U(i, j, k)$. There are some similar methods, such as differential transformation method $[4,5,11,12,22,23,13,20,21]$. Other similar schemes can be seen in $[14,15,16,17,18,19]$.

\section{Three-Dimensional Differential Transform}

Consider a function of two variables $w(x, y, t)$, and suppose that it can be represented as a product of two single-variable functions, i.e., $w(x, y, t)=$ $\varphi(x) \phi(y) \psi(t)$. Then the function $w(x, y, t)$ can be represented as

$$
w(x, y, t)=\sum_{i=0}^{\infty} \sum_{j=0}^{\infty} \sum_{k=0}^{\infty} W(i, j, k) x^{i} y^{j} t^{k} .
$$

where $W(i, j, k)$ is called the spectrum of $w(x, y, t)$. Now we introduce the basic definitions and operations of three-dimensional DT as follows[22].

Definition 2.1. Given a $w$ function which has three components such as $x, y, t$. Three-dimensional differential transform of $w(x, y, t)$ is defined

$$
W(i, j, k)=\frac{1}{i ! j ! k !}\left[\frac{\partial^{i+j+k}}{\partial x^{i} \partial y^{j} \partial t^{k}} w(x, y, t)\right]_{x=x_{0}, y=y_{0}, t=t_{0}},
$$

where the spectrum function $W(i, j, k)$ is the transformed function, which is also called the T-function. let $w(x, y, t)$ as the original function while the uppercase 
$W(i, j, k)$ stands for the transformed function. Now we define The differential inverse transform of $W(i, j, k)$ as follows:

$$
w(x, y, t)=\sum_{i=0}^{\infty} \sum_{j=0}^{\infty} \sum_{k=0}^{\infty} W(i, j, k)\left(x-x_{0}\right)^{i}\left(y-y_{0}\right)^{j}\left(t-t_{0}\right)^{k} .
$$

Using Eq. (9) in (10), we have

$$
\begin{gathered}
w(x, y, t)=\sum_{i=0}^{\infty} \sum_{j=0}^{\infty} \sum_{k=0}^{\infty} \frac{1}{i ! j ! k !}\left[\frac{\partial^{i+j+k}}{\partial x^{i} \partial y^{j} \partial t^{k}} w(x, t)\right]_{x=x_{0}, y=y_{0}, t=t_{0}} x^{i} y^{j} t^{k} \\
=\sum_{i=0}^{\infty} \sum_{j=0}^{\infty} \sum_{k=0}^{\infty} W(i, j, k) x^{i} y^{j} t^{k} .
\end{gathered}
$$

Now we give the fundamental theorem for the three-dimensional case of DTM by using the following theorem

Theorem 2.1. Assume that $W(i, j, k), U(i, j, k)$ and $V(i, j, k)$ are the differential transforms of the functions $w(x, y, t), u(x, y, t)$ and $v(x, y, t)$, respectively; then:

$$
\begin{aligned}
& \text { 1- If } w(x, y, t)=u(x, y, t) \pm v(x, y, t) \text {, then } W(i, j, k)=U(i, j, k) \pm V(i, j, k), \\
& \text { 2- If } w(x, y, t)=c u(x, y, t), \text { where } c \in \mathbf{R}, \text { then } W(i, j, k)=c U(i, j, k) \\
& \text { 3- If } w(x, y, t)=\frac{\partial}{\partial t} u(x, y, t), \text { then } W(i, j, k)=(k+1) U(i, j, k+1) \\
& \begin{aligned}
4-\text { If } w(x, y, t) & =\frac{\partial^{r+s+m}}{\partial x^{r} \partial y^{s} \partial t^{m}} u(x, y, t), \text { then } \\
& W(i, j, k)=\frac{(i+r) !(j+s) !(k+m) !}{i ! j ! k !} U(i+r, j+s, k+m) .
\end{aligned}
\end{aligned}
$$

Proof. See [22].

\section{Reformulation of the Problem}

In this section, we convert the problem (1)-(7) into a system of first order, linear, ordinary differential equation.

In Eqs. (1)-(7), the functions $f(x, y, t), p(x, y), g(x, y), h(x, t), r(y, t)$ and $m(t)$ generally are not polynomials. We assume that these functions are polynomial or they can be approximated by polynomials to any degree of accuracy. 
Then if we suppose that

$$
\left\{\begin{array}{l}
f(x, y, t) \simeq \sum_{i=0}^{n} \sum_{j=0}^{n} \sum_{k=0}^{n} F(i, j, k) x^{i} y^{j} t^{k} \\
p(x, y) \simeq \sum_{i=0}^{n} \sum_{j=0}^{n} P(i, j) x^{i} y^{j} \\
g(x, y) \simeq \sum_{i=0}^{n} \sum_{j=0}^{n} G(i, j) x^{i} y^{j} \\
h(x, t) \simeq \sum_{i=0}^{n} \sum_{k=0}^{n} H(i, k) x^{i} t^{k} \\
r(y, t) \simeq \sum_{j=0}^{n} \sum_{k=0}^{n} R(i, k) y^{j} t^{k} \\
m(t) \simeq \sum_{k=0}^{n} M(k) t^{k} .
\end{array}\right.
$$

Therefore we consider approximate solution of the form

$$
U_{n}(x, y, t)=\sum_{i=0}^{n} \sum_{j=0}^{n} \sum_{k=0}^{n} U(i, j, k) x^{i} y^{j} t^{k}
$$

The $U_{n}(x, y, t)$ is the approximation of $u(x, y, t)$. It means that

$$
\lim _{n \rightarrow \infty} U_{n}(x, y, t)=u(x, y, t)
$$

If we find the values of $U(i, j, k)$, for $i, j, k=0,1,2, \ldots, n$, then $U_{n}(x, y, t)$ can be found by using Eq. (13). To find these unknowns, we proceed as follows.

Firstly, by utilizing Theorem 2.1 and Eqs. (12) ,(13) into

$$
u_{t}-\alpha u_{x x}-\beta u_{y y}=f(x, y, t)
$$

we get

$$
\begin{aligned}
& (k+1) U(i, j, k+1)-\alpha(i+1)(i+2) U(i+2, j, k) \\
& \quad-\beta(j+1)(j+2) U(i, j+2, k)=F(i, j, k), i, j, k=0,1,2, \ldots, n .
\end{aligned}
$$

consider the initial condition

$$
u(x, y, 0)=p(x, y)
$$

By using Eq. (12)-(13) to the above equation we have

$$
\sum_{i=0}^{n} \sum_{j=0}^{n} U(i, j, 0) x^{i} y^{j}=\sum_{i=0}^{n} \sum_{j=0}^{n} P(i, j) x^{i} y^{j}, i, j=0,1, \ldots, n .
$$


then from Eq. (16), we get

$$
\begin{aligned}
& {\left[\begin{array}{cccc}
U(0,0,0) & U(0,1,0) & \ldots & U(0, n, 0) \\
U(1,0,0) & U(1,1,0) & \ldots & U(1, n, 0) \\
\vdots & \vdots & \vdots & \vdots \\
U(n, 0,0) & U(n, 1,0) & \ldots & U(n, n, 0)
\end{array}\right]_{(n+1)(n+1)}} \\
& =\left[\begin{array}{cccc}
P(0,0) & P(0,1) & \ldots & P(0, n) \\
P(1,0) & P(1,1) & \ldots & P(1, n) \\
\vdots & \vdots & \vdots & \vdots \\
P(n, 0) & P(n, 1) & \ldots & P(n, n)
\end{array}\right]_{(n+1)(n+1)}
\end{aligned}
$$

Now consider

$$
u(0, y, t)=q(y, t)
$$

By substituting Eq. (12) and (13) in above equation, we get

$$
\sum_{j=0}^{n} \sum_{k=0}^{n} U(0, j, k) y^{j} t^{k}=\sum_{j=0}^{n} \sum_{k=0}^{n} Q(j, k) y^{j} t^{k}, j, k=0,1, \ldots, n .
$$

then

$$
\left[\begin{array}{cccc}
U(0,0,0) & U(0,0,1) & \ldots & U(0,0, n) \\
U(0,1,0) & U(0,1,1) & \ldots & U(0,1, n) \\
\vdots & \vdots & \vdots & \vdots \\
U(0, n, 0) & U(0, n, 1) & \ldots & U(0, n, n)
\end{array}\right]_{(n+1)(n+1)}=\left[\begin{array}{cccc}
Q(0,0) & Q(0,1) & \ldots & Q(0, n) \\
Q(1,0) & Q(1,1) & \ldots & Q(1, n) \\
\vdots & \vdots & \vdots & \vdots \\
Q(n, 0) & Q(n, 1) & \ldots & Q(n, n)
\end{array}\right.
$$

$\mathrm{Nw}$ consider the following boundary condition

$$
u(x, 0, t)=h(x, t),
$$

By applying Eq. (12) and (13), we have

$$
\sum_{i=0}^{n} \sum_{k=0}^{n} U(i, 0, k) x^{i} t^{k}=\sum_{i=0}^{n} \sum_{k=0}^{n} H(i, k) x^{i} t^{k}, i, k=0,1, \ldots, n .
$$

then

$$
\left[\begin{array}{cccc}
U(0,0,0) & U(0,0,1) & \ldots & U(0,0, n) \\
U(1,0,0) & U(1,0,1) & \ldots & U(1,0, n) \\
\vdots & \vdots & \vdots & \vdots \\
U(n, 0,0) & U(n, 0,1) & \ldots & U(n, 0, n)
\end{array}\right]_{(n+1)(n+1)}=\left[\begin{array}{cccc}
H(0,0) & H(0,1) & \ldots & H(0, n) \\
H(1,0) & H(1,1) & \ldots & H(1, n) \\
\vdots & \vdots & \vdots & \vdots \\
H(n, 0) & H(n, 1) & \ldots & H(n, n)
\end{array}\right.
$$


And by applying Eq. (12) and (13) in

$$
u(1, y, t)=r(y, t)
$$

we have

$$
\sum_{i=0}^{n} \sum_{j=0}^{n} \sum_{k=0}^{n} U(i, j, k) y^{j} t^{k}=\sum_{j=0}^{n} \sum_{k=0}^{n} R(j, k) y^{j} t^{k}, j, k=0,1, \ldots, n .
$$

then

$$
\begin{aligned}
& {\left[\begin{array}{cccc}
\sum_{i=0}^{n} U(i, 0,0) & \sum_{i=0}^{n} U(i, 0,1) & \ldots & \sum_{i=0}^{n} U(i, 0, n) \\
\sum_{i=0}^{n} U(i, 1,0) & \sum_{i=0}^{n} U(i, 1,1) & \ldots & \sum_{i=0}^{n} U(i, 1, n) \\
\vdots & \vdots & \vdots & \vdots \\
\sum_{i=0}^{n} U(i, n, 0) & \sum_{i=0}^{n} U(i, n, 1) & \ldots & \sum_{i=0}^{n} U(i, n, n)
\end{array}\right]_{(n+1)(n+1)}} \\
& =\left[\begin{array}{cccc}
R(0,0) & R(0,1) & \ldots & R(0, n) \\
R(1,0) & R(1,1) & \ldots & R(1, n) \\
\vdots & \vdots & \vdots & \vdots \\
R(n, 0) & R(n, 1) & \ldots & R(n, n)
\end{array}\right]_{(n+1)(n+1)}
\end{aligned}
$$

Now consider the last dirichlet boundary condition

$$
u(x, 1, t)=g(x, t),
$$

By substituting Eq. (12) and (13) in above equation, we get

$$
\sum_{i=0}^{n} \sum_{j=0}^{n} \sum_{k=0}^{n} U(i, j, k) x^{i} t^{k}=\sum_{i=0}^{n} \sum_{k=0}^{n} G(i, k) x^{i} t^{k}, i, k=0,1, \ldots, n .
$$

then

$$
\left[\begin{array}{cccc}
\sum_{j=0}^{n} U(0, j, 0) & \sum_{j=0}^{n} U(0, j, 1) & \ldots & \sum_{j=0}^{n} U(0, j, n) \\
\sum_{j=0}^{n} U(1, j, 0) & \sum_{j=0}^{n} U(1, j, 1) & \ldots & \sum_{j=0}^{n} U(1, j, n) \\
\vdots & \vdots & \vdots & \vdots \\
\sum_{j=0}^{n} U(n, j, 0) & \sum_{j=0}^{n} U(n, j, 1) & \ldots & \sum_{j=0}^{n} U(n, j, n)
\end{array}\right]_{(n+1)(n+1)}
$$




$$
=\left[\begin{array}{cccc}
G(0,0) & G(0,1) & \ldots & G(0, n) \\
G(1,0) & G(1,1) & \ldots & G(1, n) \\
\vdots & \vdots & \vdots & \vdots \\
G(n, 0) & G(n, 1) & \ldots & G(n, n)
\end{array}\right]_{(n+1)(n+1)} .
$$

Finally, consider the nonlocal boundary condition

$$
\int_{0}^{b} \int_{0}^{a} u(x, y, t) d x d y=m(t)
$$

To converting this nonclassical condition to a system of equations, we use from Eq. (12) and (13) as follows:

$$
\begin{aligned}
\int_{0}^{b} \int_{0}^{a} u(x, y, t) d x d y & =\int_{0}^{b} \int_{0}^{a} \sum_{i=0}^{n} \sum_{j=0}^{n} \sum_{k=0}^{n} U(i, j, k) x^{i} y^{j} t^{k} d x d y \\
& =\sum_{i=0}^{n} \sum_{j=0}^{n} \sum_{k=0}^{n} U(i, j, k) A_{i} B_{j} t^{k} \\
& =\sum_{k=0}^{n} M(k) t^{k}
\end{aligned}
$$

where

$$
A=\left[A_{0}, A_{1}, \ldots, A_{n}\right]^{T}, \quad B=\left[B_{0}, B_{1}, \ldots, B_{n}\right]^{T},
$$

with

$$
A_{i}=\int_{0}^{a} x^{i}=\frac{a^{i+1}}{i+1}, \quad B_{j}=\int_{0}^{b} y^{j}=\frac{(b)^{j+1}}{j+1},
$$

Then from Eq. (26), we obtain

$$
\begin{aligned}
{\left[\sum_{i=0}^{n} \sum_{j=0}^{n} U(i, j, 0), \sum_{i=0}^{n} \sum_{j=0}^{n} U(i, j, 1), \ldots, \sum_{i=0}^{n} \sum_{j=0}^{n} U(i, j, n)\right] } & \\
& =\left[M_{0}, M_{1}, \ldots, M_{n}\right]
\end{aligned}
$$




\section{Determining Final Linear Equations}

In this section, to finding the values of $U(i, j, k)$, for $i, j, k=0,1,2, \ldots, n$, we must construct a system of $(n+1)(n+1)(n+1)$ equations. To this end we arrange the obtained linear equations from the previous section. Because the problems (1)-(7) have been not defined for entire of the range, we select only the equations that satisfy in the defined range.

Firstly, the $(n+1)(n+1)$ values of $U$ can be obtained from Eq. (17) as follows:

$$
U(i, j, 0)=P(i, j), i, j=0,1, \ldots, n .
$$

We find $n(n+1)$ values of $U$ from Eq. (19)

$$
U(0, j, k)=Q(j, k), j=0,1, \ldots, n, k=1,2, \ldots, n .
$$

And the $n^{2}$ values of $U$ can be given by Eq. (21) to the following case

$$
U(i, 0, k)=H(i, k), i, k=1,2, \ldots, n .
$$

We obtain $3 n^{2}+3 n+1$ values of $U$ From Eq. (28)-(30) independently. Then the $n^{3}$ remainder values of $U$ must be obtained from the other equations. We choose $n^{2}$ equations from Eq. (23)

$$
\sum_{i=0}^{n} U(i, j, k)=R(j, k), j, k=1, \ldots, n,
$$

and $n^{2}-n$ equations from Eq. (25)

$$
\sum_{j=0}^{n} U(i, j, k)=G(i, k), i=1, \ldots, n, k=2, \ldots, n .
$$

and $n$ equations from Eq. (27)

$$
\sum_{i=0}^{n} \sum_{j=0}^{n} U(i, j, k)=M_{k}, k=1,2, \ldots, n .
$$

Finally, the remainder $n^{3}-2 n^{2}-n$ equations can be found from Eq. (15) by 
the following equations

$$
\begin{aligned}
& U(i, j, k+1)=\frac{1}{(k+1)}(\alpha(i+1)(i+2) U(i+2, j, k) \\
& \quad+\beta(j+1)(j+2) U(i, j+2, k)+F(i, j, k)) \\
& i, j=1,2, \ldots, n-2, k=0,1,2, \ldots, n, \\
& U(0, j, k+1)=\frac{1}{(k+1)}(\alpha 2 U(2, j, k)+\beta(j+1)(j+2) U(0, j+2, k)+ \\
& \quad F(0, j, k)), j=1,2, \ldots, n-2, k=0,1,2, \ldots, n-1, \\
& U(i, 0, k+1)=\frac{1}{(k+1)}(\alpha(i+1)(i+2) U(i+2,0, k)+\beta 2 U(i, 2, k) \\
& +F(i, 0, k)), i=1,2, \ldots, n-2, k=0,1,2, \ldots, n-1,
\end{aligned}
$$

Eqs. (31)-(34) give a linear algebraic system of $n^{3}$ equations that by solving this system, the remainder values of $U$ will be obtained. Now from Eq. (13), we can obtained $U_{n}(x, y, t)$ that it is the approximation of $u(x, y, t)$.

\section{Conclusions}

In this article, the solution of the second order two space dimensional diffusion equation has been discussed by using differential transformation method. Converting the model of partial differential equation to a system of linear equations, is the main part of this paper. The computational difficulties of the other methods can be reduced by applying this process.

\section{References}

[1] B. Soltanalizadeh, Numerical analysis of the one-dimensional Heat equation subject to a boundary integral specification, Optics Communications, 284 (2011) 2109-2112

[2] L. I. Kamynin, A boundary value problem in the theory of the heat conduction with nonclassical boundary condition, Z. Vychisl. Mat. Fiz., 4 (1964) 1006-1024.

[3] N. I. Ionkin, Solutions of boundary value problem in heat conductions theory with nonlocal boundary conditions, Differents. Uravn., 13 (1977) 294-304. 
[4] A. Borhanifar, R. Abazari, Numerical study of nonlinear Schrödinger and coupled Schrdinger equations by differential transformation method, Optics Communications 283 (2010) 2026-2031.

[5] R. Abazari, A. Borhanifar, Numerical study of the solution of the Burgers and coupled Burgers equations by a differential transformation method, Comput. Math. Appl., 59 (2010) 2711-2722.

[6] SA. Beilin, Existence of solutions for one-dimensional wave equations with nonlocal conditions. Electron J Diff Eqn., 76 (2001) 1-8.

[7] IS. Gordeziaini, GA. Avalishvili, On the constructing of solutions of the nonlocal initial boundary problems for one-dimensional medium oscillation equations. Matem Modelirovanie 12(1) (2000) 94-103.

[8] JR. Cannon, The solution of the heat equation subject to the specification of energy. Quart Appl Math. 21 (1963) 155-60.

[9] J. G. Batten, Second-order correct boundary conditions for the numerical solution of the mixed boundary problem for parabolic equations. Math Comput. 17 (1963) 405-13.

[10] H.R. Ghehsareh, B. Soltanalizadeh,S. Abbasbandy, A matrix formulation to the wave equation with nonlocal boundary condition, Inter. J. Comput. Math., 88 (2011) 1681-1696.

[11] B. Soltanalizadeh, Differential Transformation method for solving onespace-dimensional Telegraph equation, comput. appl. math., 30(3) (2011) 639-653.

[12] B. Soltanalizadeh, M. Zarebnia, Numerical analysis of the linear and nonlinear Kuramoto-Sivashinsky equation by using Differential Transformation method, Inter. J. Appl. Math. Mechanics, 7(12) (2011) 63-72.

[13] B. Soltanalizadeh, A. Yildirim, Application of Differential Transformation method for numerical computation of Regularized Long Wave equation, Z. fuer Naturforschung A., 67a (2012) 160-166.

[14] H. Roohani Ghehsareh, S. Abbasbandy, B. Soltanalizadeh, Analytical solutions of the slip MHD viscous flow over a stretching sheet by using the Laplace Adomian Decomposition Method, Zeitschrift fuer Naturforschung A., Zeitschrift fuer Naturforschung A., 67a, (2012) 248-254. 
[15] B. Babayar, B. Soltanalizadeh, Numerical solution for system of singular nonlinear volterra integro-differential equations by Newton-Product method, Applied mathematics and computation, 219 (2013) 8375-8383.

[16] B. Soltanalizadeh, HR Ghehsareh, A. Yldrm, S. Abbasbandy, On the Analytic Solution for a Steady Magnetohydrodynamic Equation, Zeitschrift fuer Naturforschung A., 68a (2013) 412-420.

[17] B. Babayar, B. Soltanalizadeh, Numerical solution of nonlinear singular Volterra integral system by the Newton-Product integration method, Mathematical and computer modelling, 58 (2013) 1696-1703.

[18] B. Soltanalizadeh, H.R. Ghehsareh, S. Abbasbandy, Development of the tau method for the nu- merical study of a fourth-order parabolic partial differential equation, U.P.B. Sci. Bull., Series A, 75 (2013) 165-176.

[19] B. Soltanalizadeh, An Approximation Method Based on Matrix Formulated Algorithm for the Numer- ical Study of a Biharmonic Equation, Australian Journal of Basic and Applied Sciences, 5(12) (2011) 501-506.

[20] B. Soltanalizadeh, Application of Differential Transformation method for solving a fourth-order parabolic partial differential equations, International journal of pure and applied mathematics, 78 (3) (2012) 299-308.

[21] B. Soltanalizadeh, Application of Differential Transformation Method for Numerical Analysis of Kawa- hara Equation, Australian Journal of Basic and Applied Sciences, 5(12) (2011) 490-495.

[22] F. Ayaz, Solutions of the systems of differential equations by differential transform method, Appl. Math. Comput., 147 (2004) 547-567.

[23] R. Abazari, M. Ganji, Extended two-dimensional DTM and its application on nonlinear PDEs with proportional delay, Int. J. Comput. Math., 88 (2011) 1749-1762.

[24] I. Hashim, Comparing Numerical Methods for the Solutions of TwoDimensional Diffusion with an Integral Condition., Appl. Math. Comput., 181 (2006) 880-885.

[25] M. Siddique, Smoothing of Crank-Nicolson Schemes for the TwoDimensional Diffusion with an Integral Condition, Appl. Math. Comput., 214 (2009) 512-522. 
[26] A. B. Gumel, W. T. Ang and E. H. Twizell, Efficient Parallel Algorithm for the Two Dimensional Diffusion Equation Subject to Specification of Mass, Intern. J. Computer Math., 64 (1997) 153-163.

[27] M. Siddique, A Comparison of Third Order $L_{0}$-Stable Numerical Schemes for the Two-Dimensional Homogeneous Diffusion Problem Subject to Specification of Mass, Appl. Math. Sciences, 4(13), (2010) 13 611-621.

[28] A. Murthy, J. Verwer, Solving parabolic integro-differential equations by an explicit integration method. J Comput Appl Math., 39 (1992) 121-32.

[29] G. Dagan, The significance of heterogeneity of evoling scales totrnasport in porous formations, Water Resour. Res. 13 (1994) 3327-3336.

[30] W. A. Day, Parabolic equations and thermodynamics. Quart Appl Math 50 (1992) 523-533.

[31] M. Siddique, Numerical computation of two-dimensional diffusion equation with nonlocal boundary conditions, IAENG Inter. J. Appl. Math.,4-1-04.

[32] M. Dehghan, Implicit locally one-dimensional methods for two-dimensional diffusion with a nonlocal boundary condition, Math. And Computers in simulation 49 (1999), 331349.

[33] A. B. Gumel, W. T. Ang and E. H. Twizell, Efficient Parallel Algorithm for the Two Dimensional Diffusion Equation Subject to Specification of Mass., Intern. J. Computer Math, Vol. 64 (1997) 153-163. 
